\title{
Research on the Development Status of International Fashion Design and Engineering Major in the Information Technology Age
}

\author{
Jun YIN ${ }^{1,2, *}$ \\ ${ }^{1}$ Wuhan Textile University, Wuhan, 430073, China \\ ${ }^{2}$ China Academy of Art, Hangzhou ,310014, China
}

12408113@qq.com

Keywords: Clothing design and engineering; smart clothing; information interaction technology; big data

\begin{abstract}
Considering the research findings of international fashion design and engineering major from 2014 to 2017, there are four obvious trends. Firstly, the research of smart clothing shows clearly upward trend, smart clothing design is generally divided into two categories: the common health monitoring clothing and disease monitoring clothing. However, there are still many disadvantages in the existing products. Secondly, the number of functional clothing design findings is second to intelligent clothing design; besides, it is higher than any other types of design research. The current research focuses on two aspects: the positioning of functional clothing design and product technology implementation. Thirdly, with the rapid breakthrough trend of information interaction technology, the original mode of clothing product design, production, sales, management and other departments has changed. The main achievements are as follows: 3D simulation (3D) and clothing virtual fitting, VR virtual reality technology and clothing e-commerce, two-dimensional code virtual sales mode, etc.. Fourthly, with the help of big data network and advanced computer programming design, more research makes in-depth analysis on contemporary clothing consumption behavior and consumers' psychology and demand, which accordingly helps formulate scientific and systematic clothing marketing strategy and management program.
\end{abstract}

\section{Introduction}

The rapid development of information technology not only urge the designers and researchers to use latest technology to design better clothing, but also brings the rapid integration of different disciplines, which can provide an opportunity for people to get a better life. In this time, most research of clothing design mainly focuses on four aspects. [1]They are smart clothing consumers and product positioning, the product design and technology implementation of functional clothing, the application of information interaction technology on clothing design, clothing marketing; and the design and development of clothing computer assisted system under the background of big data.

\section{The consumer positioning and function design of smart clothing and product}

Researches on smart clothing have shown a significant upward trend from 2014 to 2017(Figure 1), but the current research results show that smart wearable devices still have different shortcomings, such as not comfortable for long-time wearing, not accurate and other issues(Min Chen \& Chin-Feng Lai, Huazhong University of Science and Technology, National Chung Cheng University in Taiwan, 2016). At present, the research and development of smart clothing are still in the early stage, and there are still many problems needed to be solved. From the perspective of product design, there is a gap between assessment standard of fashion designer and consumer. For example, consumers treat "function" as the most important demand, at the same time, "seamless stitching”, "easy-using”, "cost-effective", "attractiveness", "comfortableness" are also their important purchase criteria (Ann Perry, Laura Malinin. etc. Colorado State University, 2017). [2] The positioning of specific consumers needs to be more detailed, which means it can be multilevel 
classified according to consumers' age, gender, health needs, disease characteristics, in particular, the design criteria should be ergonomically correct.

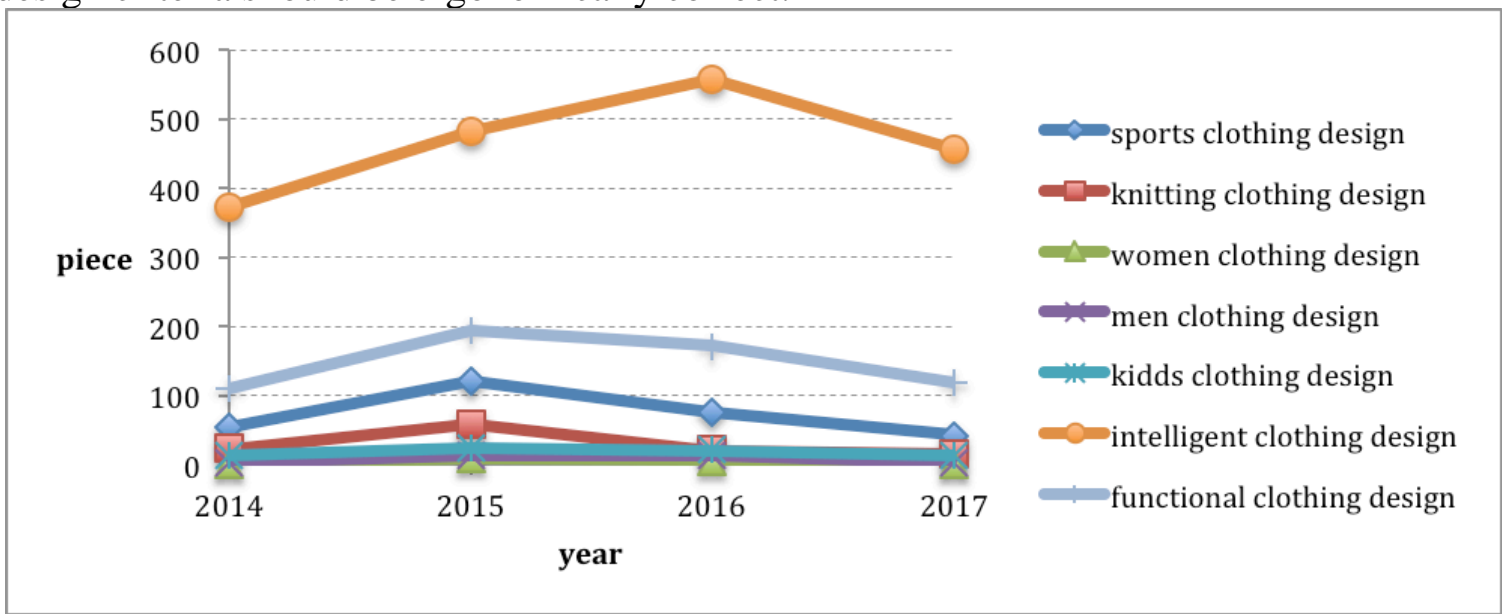

Figure 1 Statistical analysis of published papers on smart clothing design and development (searching results from SCIENCEDIRECT 2010-2017)

Based on the prominent functional characteristics of intelligent technology, the target consumer groups have more clear functional design requirements. [3] Smart clothing design is generally divided into two categories: (1) general health monitoring clothing. In the big data age, the design and development of health monitoring clothing has been integrated with medical emergency response, emotional care, disease diagnosis and real-time interaction. In particular, the ECG signals collected by smart clothing are used for emotion monitoring and detection, which can better interact with people (Min Chen \& Chin-Feng Lai, Huazhong University of Science and Technology, National Chung Cheng University in Taiwan,2016). (2) Disease monitoring clothing. This kind of smart clothes can detect changes of human body by considering pressure, temperature and gas, which can finally help patients to know their health condition change in time. Different from health monitoring clothing, the disease monitoring clothing is more targeted. For instance, e-nose system can detect gas from the nose and skin and then provide useful information, such as early diagnosis, comfort, continuous self monitoring, and finally reduce medical expense (Seesaad, etc,2015). [4]After the operation, the smart monitoring clothes which breast cancer patients wear can provide convenience for the operation nursing (Karen L. LaBat, University of Minnesota, 2016).

\section{The product design and technical implementation of functional clothing}

It can be seen from Figure 2 that in the past 2014-2017 years, the research results number of functional clothing design is second only to the smart clothing design. The current research focuses mainly on two aspects:

\section{The positioning of functional clothing design}

There are two categories of functional clothing design: general functional clothing and special functional clothing. Consumers of general functional clothing are usually healthy people, their sustained attention to their own health and their prevalence of outdoor sports have promoted the continuous growth of sporting goods and outdoor functional clothing sales. The main consumers of special functional clothing are special occupation workers and special disabled people. For example, the functional clothing developed for professional athletes has the function of improving people's sports performance and helping athletes better exert their professional skill. [5] Chupo Ho and Yuhan Au from Hong Kong Polytechnic University designed a functional vest which was served for professional rowers in rowing sports. This design is customized by several elements, such as, 3D scanning technology, personal preference orientation, and personal health characteristics. It can also provide personalized customization service including fabric types and fashion style and craft details. It aims for reducing material wear as well as helping athletes to quickly evaporate their heat consumption. There are also protective surgical gowns designed to improve the success rate of 
surgery based on the theory of thermal and ergonomics (O. Troynikov, N. Nawaz, C. Watson, Royal Melbourne Institute of Technology University, Australia, 2014). The researchers have pointed out that most of fashion design and research is for healthy and capable consumers, while the needs of the special disabled population are not satisfied (Beecher \& Paquet, 2005; Newell \& Cairns, 1993). In recent years, the differentiation of functional clothing is more and more obvious. For example, functional clothing product design is more and more inclined to solve the problems in life and work, improve the quality of life, and reduce the pain or inconvenience caused by disease and physiological deficiency. Research team of the Hades Phil University developed a functional vest for the child cancer patients who need long-term medical care. This vest can reduce the complications caused in the process using central venous catheter (2017). Researcher Helen s. Koo from University of California at Davis and Xiao Huang from Auburn University cooperated for applying light emitting diode into bicycle clothing design. The experiments show that the luminescence on rider's lower body can be more conducive to the identification device at night than that of the upper body. Researchers Megan Johnston and Helen Koo from University of California at Davishave studied the satisfaction and preference of women's climbing sports apparel, then they proposed the idea of using different materials design in different parts (2016). Fred Han, Kristina Shin\& Daniel Chow (2016) from Hong Kong Polytech University developed a scuba diving suit which can provide water medical care service for disabled people.

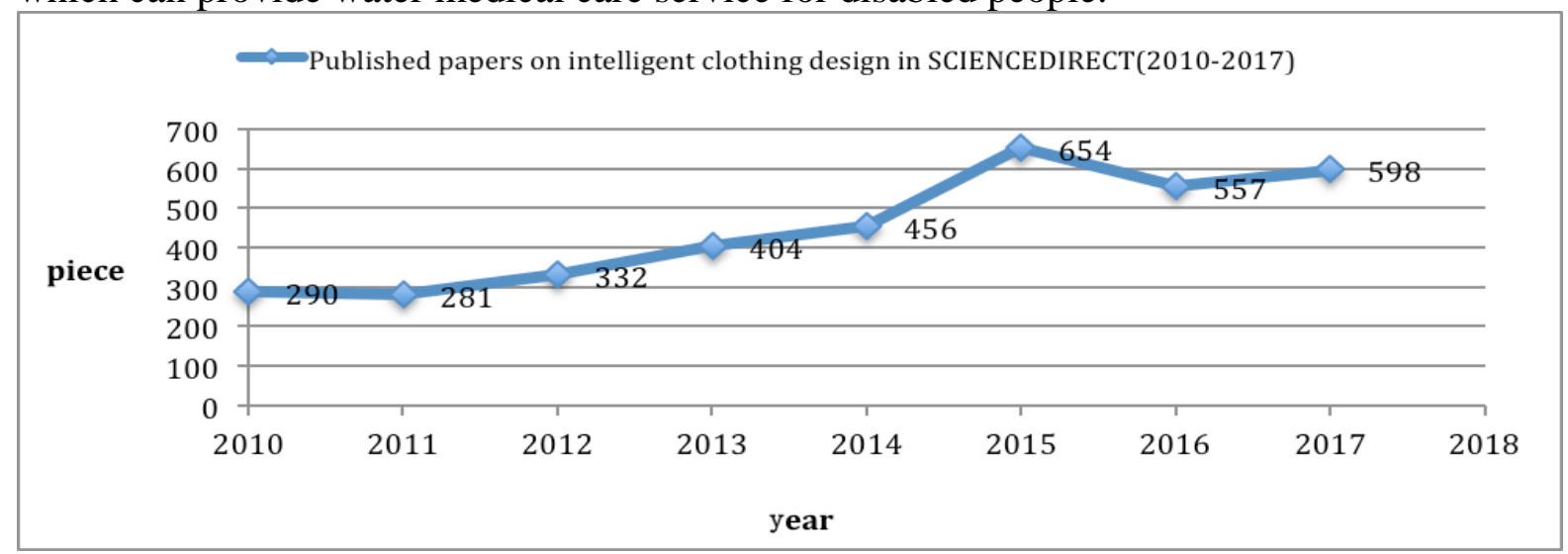

Figure 2 Classified statistics of papers on "fashion design" from SCIENCEDIRECT

\section{Technology implementation of functional clothing product}

There are different requirements for different functional clothing, and these requirements can be satisfied by designing different style and using different materials. For example, in a study of reflective winter clothing study, researchers found that using reflective insulation layer can increase the cold resistance; using low radiation metal coating is an important way to improve the usefulness of cold weather clothing (Matthew Patrick Morrissey, 2014). In another study, researchers found that the use of touch screen gloves increased with the worldwide popularity of Smartphone. Usually, the technical solution of touch screen gloves is preventing the current from the skin to the touch screen and reducing the electrical conductivity. In this way, the screen functionality can be achieved.

\section{Application of interactive technology in fashion design and fashion marketing}

Clothing is one of industries with the highest degree of globalization and the fastest change. With the continuous development of high-tech, the original production patterns of various departments of garment product design, production, sales, management have all changed.

\section{Three dimensional simulation (3D) and garment virtual fitting.}

The rapid development of e-commerce has caused clothing online shopping become the main way of consumption. According to Cowen\&Co data, in 2017, Amazon apparel products sales will reach $\$ 28$ billion, and it will become the largest clothing retailer in the United States. The current 
3D virtual simulation technology can transform the 2D mode into 3D mode, and create close distance visualization of main product. However, the current technology still exists "distortion" problem, that is, there is a certain difference between the actual and virtual clothing (Eunyoung Lee \& Huiju Park, Cornell University, 2016). Hence, Kaixuan Liu from Xi'an Polytechnic University and Edwin Kamalha from an university in Leo, France have proposed a garment fitness evaluation model based on Naive Bayes model (2017). The input of this model is the digital clothing pressure of different boy parts produced by the 3D garment CAD software, and the output is the prediction result of the fitness (fit or not fit) of the garment. The automatic clothing fitting system can adapt the garment model to the target human body. Cloth simulation techniques can bring costumers the natural and realistic fitting feeling. But the process and efficiency of 3D garment simulation may vary according to different clothing, body model and fabric type, which in turn may influence the effect of virtual fitting(Anne Porterfield \& Traci A. M. Lamar,2016). Therefore, in addition to the 3D virtual fitting technology, researchers have developed a new evaluation method: clothing fitting evaluation (Yueh-Ling Lin, Taiwan's National Tsing Hua University,2015). This method enhances the interaction between the fitting person and the virtual fitting system, and helps to make up for the lack of system development at the present stage.

\section{VR virtual reality technology and clothing e-commerce}

The year 2016 is known as the VR technology era, VR technology have many features such as multisensory, prominent presence, real-time interaction, etc. For clothing e-commerce, VR technology can realize the virtual fitting, reduce production costs, change consumer behavior patterns, and ultimately change the existing clothing production chain and supply chain system. Smart store technology (SIST) is rapidly developing in the retail sector, such as the appearance of tablet PCs, Smartphone, augmented reality, virtual commodity catalogs and smart tags, which are all changing the shopping experience (Spears,2014). But at present, several parts of the research of VR technology are still not mature, such as simulated environment, perception, natural skills and sensing equipment, and there's still a long way to go to actually achieve commercial application.

\section{Two dimensional code virtual sales}

Two dimensional codes has been considered as a technology that can completely change the retail business. Based on the positioning service and augmented reality sense, it has multidimensional attributes and mobile commerce service feature (Shin \& Chang,2014). Researches of Eun Young Kim and Namhee Yoon from Incheon National University have shown that extensive use of two-dimensional code technology is conducive to the creation of virtual retail market and the solutions of constructing intelligent business.

\section{Design and development of clothing computer aided system in the era of big data}

With the help of data network and advanced computer programming design, researchers make in-depth analysis of contemporary clothing consumption behavior, explore consumers' psychology and demand, and then formulate a scientific and systematic marketing strategy and managing programs.

\section{Product recommendation system}

Through a comprehensive survey of clothing recommendation system, four types of systems are classified according to their key functions: clothing search/retrieval, clothing recommendation, fashion coordination and intelligent recommendation system. The first two products work based on the similarity between the features of the new product and the history of the previously used product or wardrobe. The latter two models use different styles and have their own characteristics in the process of style learning. Many recent studies have focused on the system model which use a variety of machine learning algorithms, such as neural networks and potential support vector machines (SVM), etc. (Congying Guan, Shengfeng Qin \& Wessie Ling, Northumbria University). However, researchers Wei-Lun Chang, Osmud Rahman from Tamkang University and Ryerson 
University have suggested choosing the clothing recommendation system which analyze consumers' image and then give purchasing suggestion (2015). Another way is to do statistical analysis of individual network consuming behavior and sort it as consumers' individualized data by applying "perceptual technology" (e-marketer,2015). This can provide the basic data for marketers to judge the market trend. Indian scholars proposed an updated method, that is, through the analysis of gait entropy image to identify the shape and dress features of consumers(Anup Nandy,2016). This new perspective provides more accurate predictions of consumer recommendations. Overall, these cases show that product recommendation is a booming, creative, profitable, but unstable market.

\section{Design participation system}

The internet not only shortens the distance between producers and consumers, but also brings new ways of production for enterprises (Fred Han, Hong Kong Polytechnic University, 2016). Open innovation has become a new model for enterprises to save innovation costs and improve the success rate of innovation. In this open environment, enterprises understand the market demand, make up their own development problems from external partners, and learn valuable information from suppliers, customers, end users, and university researchers (C. Anthony Di Benedetto, 2014). All these can do more precise products positioning of target consumer. This concept, also known as "co-design", is a method of "user participation in the design process", which is recognized by both business and Ethics (Wilkinson \&amp; De Angeli, 2014).Through the collection and arrangement of the information of target consumer, the "online self customization" (OSC) model has become one of the important structural changes in the fashion industry production mode. When the online customization system analyzes consumers' behavior, it would put forward the corresponding customized product identification (identity) and identification code. After that, it will solve the problem of consumers' self uncertainty and meet their self-satisfaction demand by analyzing consumer's cognitive development and emotional identity(Wolter \&amp; Cronin, 2016).Based on the intrinsic motivation of social identity theory and identity culture, this study establishes a consumer defined product identification model (Soyeon Kwon, Ph.D, 2017).

\section{Management decision system}

In the product development process, the application of consumers' clothing need and expectation system model could filter the big data, then analyze the demography, psychological characteristics, life style and technical characteristics, which can identify target customers' expectations of garment and determine the relevant characteristics of products in the sales process (Laurel D. Romeo Young-A \&amp; Lee, 2016). The comparative data of clothing production and sales provide the basis for establishing the decision support system of garment production outsourcing. Using fuzzy inference method, this system making modeling of the illogical global outsourcing decision evaluation model, so that it can solve the complexity and uncertainty in the global outsourcing process. In addition, it can also improve the production efficiency of enterprises, change the experience-dependent judgment in the decision-making process, and put the rational analyzing conclusions as the basis (Asli Aksoy, 2015).

\section{Acknowledgements}

In this paper, the research was sponsored by Teaching research project of Wuhan Textile University in 2017 (2017JY012); College Students' innovation and entrepreneurship training program of Wuhan Textile University in 2017(2017CXCY029).

\section{References}

[1] Erin Lawless, Katalin Medvedev. Assessment of sustainable design practices in the fashion industry: experiences of eight small sustainable design companies in the Northeastern and Southeastern United States [J] -International Journal of Fashion Design, Technology and Education, 
2016, 9 (1): 41-50

[2] Louise Lundblad, Iain A. Davies. The values and motivations behind sustainable fashion consumption [J] -Journal of Consumer Behaviour , 2016, 15: 149-162

[3] Marsha A. Dickson, Rita K. Chang . Apparel Manufacturers and the Business Case for Social Sustainability [J] -The Journal of Corporate Citizenship Issue. 2015,55-72

[4] Huantian Cao, Marsha A. Dickson, Kelly Cobb, Martha Carper, Crescent Scudder, Crispin Wong. Applying a sustainability performance measurement tool in designing and developing automotive employee uniforms [J]-International Journal of Fashion Design, Technology and Education, 2015, 8(2): 78-86

[5] Miguel Angel Gardetti, Subramanian Senthilkannan Muthu. Sustainable apparel Is the innovation in the business model? -The case of IOU Project [J]-Textiles and Clothing Sustainability, 2015,1 (1) :1-9 